\title{
Interlaminar Flux Density Distribution at Joints of Overlapping Stacked Electrical Steel and Amorphous Ribbons
}

\author{
Sezer Erdem* and Naim Derebasi \\ Department of Physics, Uludag University, 16059 Gorukle, Bursa, Turkey
}

(Received 6 August 2010, Received in final form 22 September 2010, Accepted 28 September 2010)

\begin{abstract}
The design of joints in a transformer core significantly affects the transformer's efficiency. Air gaps cause variations in the flux distribution at the joints of the laminations, which depend on the geometry. Two similar samples consisting of electrical steel strips and amorphous ribbons were made. The spatial flux distributions were determined using an array of search coils for each sample. 2D models of these samples were created and examined by finite element analysis. The magnetic flux distribution for each lamination in the samples was computed. The results show that the flux density in amorphous ribbons above and below the air gap starts to approach saturation at lower flux density levels than for electrical steel. The flux density measured using the search coil under the air gap is increased in amorphous ribbons and decreased in the electrical steel with increasing frequency.
\end{abstract}

Keywords : electrical steel, amorphous ribbon, 2D finite element analysis, flux density distribution

\section{Introduction}

The efficiency of a transformer core changes with the design of the joints where the yokes and limbs meet. In these regions, the flux may deviate from the rolling direction of the steel or become distorted so that local areas of high loss are produced [1]. Previous research also analyzed the normal flux distribution along the strip direction at the joints of overlapping electrical steel laminations, which simulates the flux distribution in a zip-type unicore [2].

Air gaps, which cause variation in the flux distribution, exist at the corners and in the yoke of transformer laminations, depending on the geometry used when the transformer core is manufactured. Computational methods based on the solution of Maxwell's equations, combined with a set of magnetization data measured on single sheets of material, may yield absolute predictions of performance based on previous core measurements that are independent of the design constants used. To obtain good accuracy, these methods depend on the introduction of factors representing estimated loss components resulting from the interlaminar flux distribution [2]. In this paper, we focused

*Corresponding author: Tel: +90-224-2941772

Fax: +90-224-2941899, e-mail: serdem@uludag.edu.tr on understanding the normal flux density distribution at the joint ends of stacked electrical steel strips and amorphous ribbons. Specifically, we investigated the change in flux density with changing frequency in the region near the air gap for both electrical steel and amorphous ribbons. Samples made of electrical steel or amorphous ribbons were modeled using ANSYS ${ }^{\mathrm{TM}}$. The magnetic flux densities measured from both the electrical steel strips and the amorphous ribbons were compared with the predicted values obtained from 2D finite element analysis (FEA).

\section{Experimental and Theoretical Procedures}

We investigated normal flux transitions among electrical steel laminations and amorphous ribbons separately. For this purpose, we prepared two similar samples made of electrical steel or amorphous ribbons. The electrical steel model consisted of four electrical steel laminations designated 1 to 4, as shown in Fig. 1. The amorphous ribbon model was similar except for an important difference: In the amorphous model, each ribbon (1 to 4) consisted of five overlapping ribbons packed as one. The effect of air gaps on the flux density distribution in these samples was investigated. Search coils (X, Y, and Z) wound around the laminations were placed as shown in Fig. 1, and magnetic induction measurements for each sample were made using 


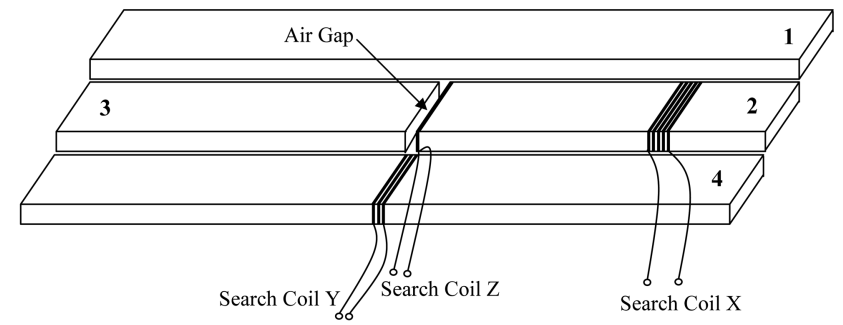

Fig. 1. Experimental samples prepared using electrical steel and amorphous ribbons.

the measuring system shown in Fig. 2.

The laminations consisted of grain-oriented $3 \% \mathrm{SiFe}$ M4 electrical steel strips $(0.27 \mathrm{~mm}$ thick). The ribbon model used Metglas-2605SC $\left(\mathrm{Fe}_{81} \mathrm{~B}_{13.5} \mathrm{Si}_{3.5} \mathrm{C}_{2}\right)$ amorphous ribbons (30 $\mu \mathrm{m}$ thick). The locations and turn numbers of search coils $\mathrm{X}, \mathrm{Y}$, and $\mathrm{Z}$ were the same in both models. The calculations and experimental flux density measurements were made under sinusoidal flux excitation. The electrical steel laminations were magnetized over a frequency range from 50 to $400 \mathrm{~Hz}$ at peak flux density levels from 0.1 to $1.5 \mathrm{~T}$. The amorphous ribbons were magnetized over a frequency range from 50 to $400 \mathrm{~Hz}$ at peak flux density levels from 0.1 to $1.0 \mathrm{~T}$, owing to limitations of the magnetizing circuit.

In the strip-wound material core, the overlapping areas and air gap are very important, and the magnetic flux is not confined to one layer but circulates through several layers. Therefore, if the air gap and overlapping areas are not considered, the calculated and measured peak magnetic flux density results will differ. A 3D analysis can be used to obtain the solution, but this is unnecessary because of the $2 \mathrm{D}$ symmetry of the magnetic path. A $2 \mathrm{D}$ analysis is more practical for this engineering application [3], so a 2D FEA was used to investigate the change in magnetic flux transition for the overlapping stacked samples. The samples were modeled using ANSYS ${ }^{\mathrm{TM}}$, and the properties of different materials, such as magnetic permeability and B-H curves, were defined in the models. A free triangular mesh, including mesh refinement around the air gap to improve the results, was set up with 13,233 nodes and 6,592 elements for the electrical steel model, and 13,337 nodes and 6,644 elements for the amorphous ribbons. For the electrical steel laminations, the ANSYS ${ }^{\mathrm{TM}}$ modeling and mesh refinement close to the air gap are shown in Fig. 3. A 2D static magnetic analysis and vector potential
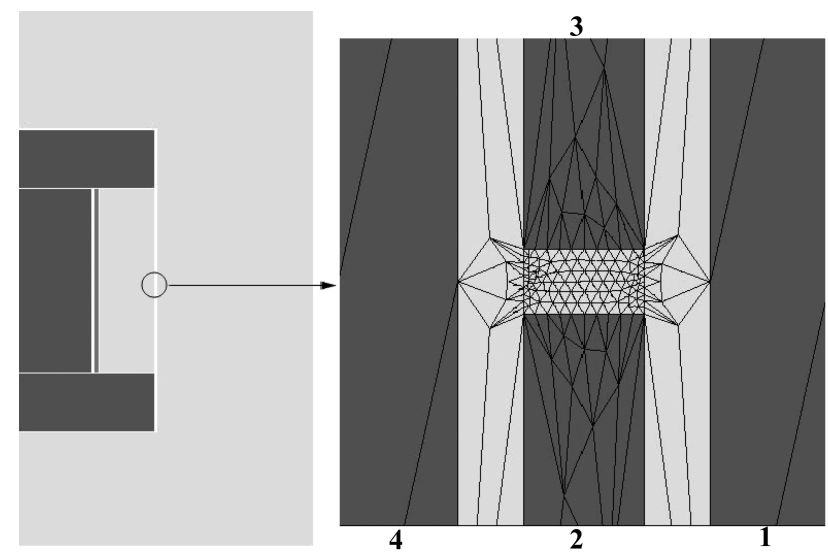

Fig. 3. Overall geometry and mesh refinement close to the air gap for electrical steel laminations.

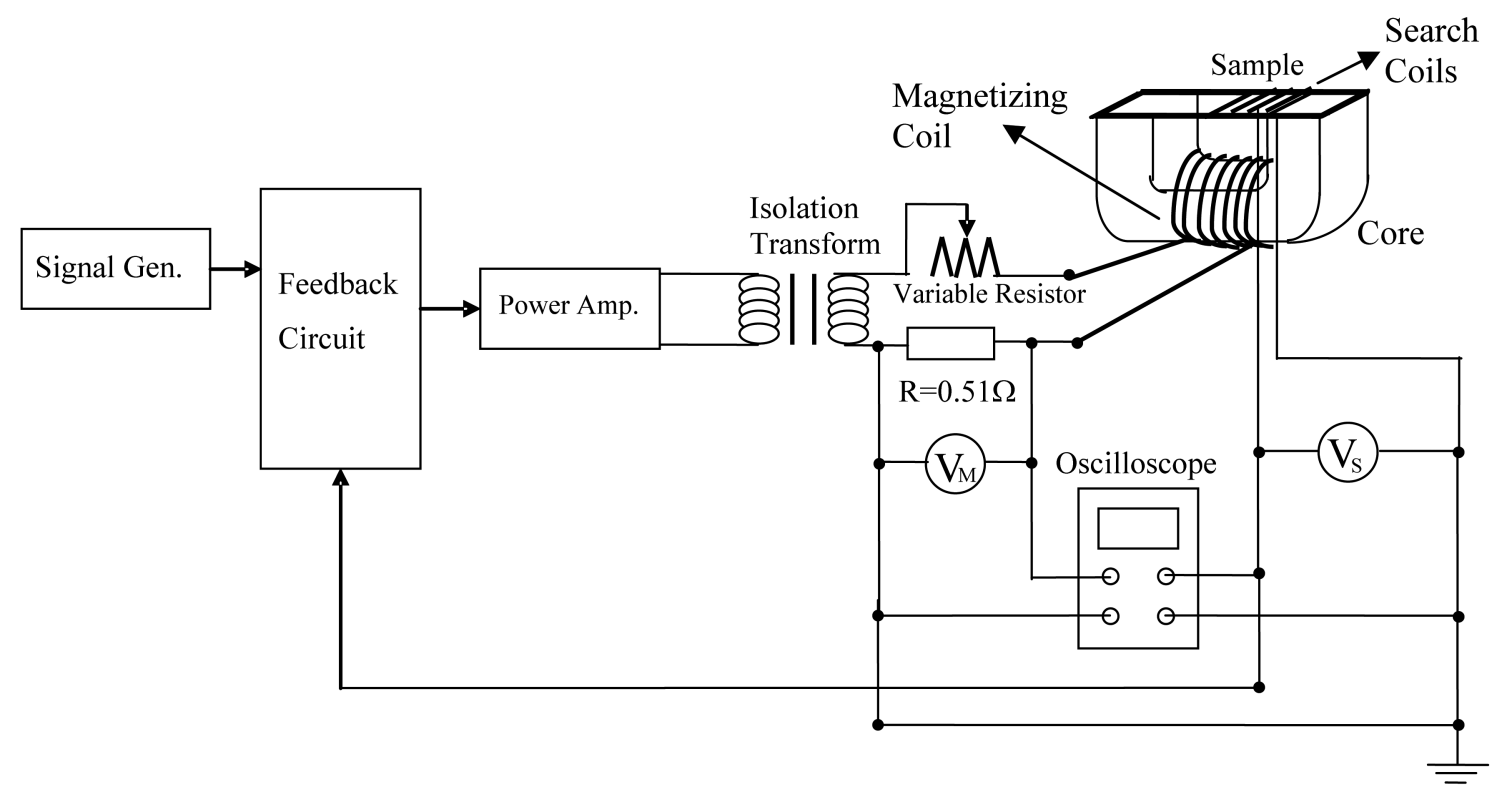

Fig. 2. Measuring system. 
formulation in one direction were used to solve the problem. The magnetic flux distribution for each lamination in the samples was computed. The basic B-H characteristics needed as input data for the problem were measured experimentally.

\section{Results and Discussion}

Fig. 1 shows the positions of search coils on laminations 1 to 4 . The reference experimental flux density was calculated from the emf induced in search coil X wound on lamination 2. The flux in lamination 2 drops rapidly when it approaches the air gap between laminations 2 and 3 , whereas the flux increases from the nominal level to saturation in the regions opposite the air gap in laminations 1 and 4 . The flux is mainly confined to the rolling direction, but a high normal flux occurs between laminations in the joint [1]. The experimental measurements for electrical steel laminations show that in the central lamination, 2, when the flux density was set to $0.500 \mathrm{~T}$ via search coil $\mathrm{X}$, the flux increases to $0.791 \mathrm{~T}$, as indicated by search coil $\mathrm{Y}$ in lamination 4, whereas it drops dramatically to about $5 \mathrm{mT}$, as measured by search coil $\mathrm{Z}$ in the joint $(50 \mathrm{~Hz})$. The experimental measurements for amorphous ribbons show that in the central ribbon, 2, when the flux density was set to $0.500 \mathrm{~T}$ via search coil $\mathrm{X}$, the flux increases to $0.598 \mathrm{~T}$, as indicated by search coil $\mathrm{Y}$ in ribbon 4, whereas it drops to $67 \mathrm{mT}$ as measured by search coil $\mathrm{Z}$ in the joint $(50 \mathrm{~Hz})$. The variations in flux density (B) with frequency (f) in search coils $\mathrm{Y}$ and $\mathrm{Z}$ when the flux density was set to $0.500 \mathrm{~T}$ via search coil $\mathrm{X}$ are shown in Figs. 4 and 5, respectively. These measurements were also made in the flux density range from 0.1 to $1.5 \mathrm{~T}$ for the electrical steel and from 0.1 to $1.0 \mathrm{~T}$ for the amorphous ribbons. The maximum measurement errors

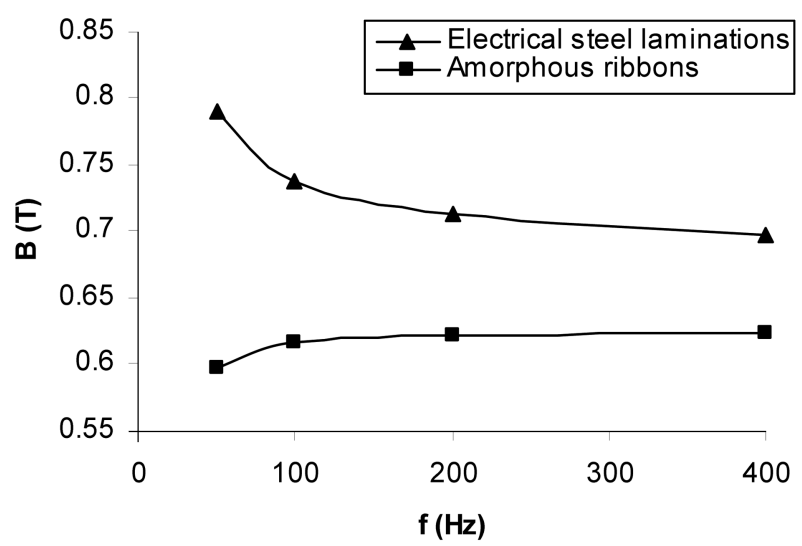

Fig. 4. Variation in flux density (B) with frequency (f) for search coil Y.

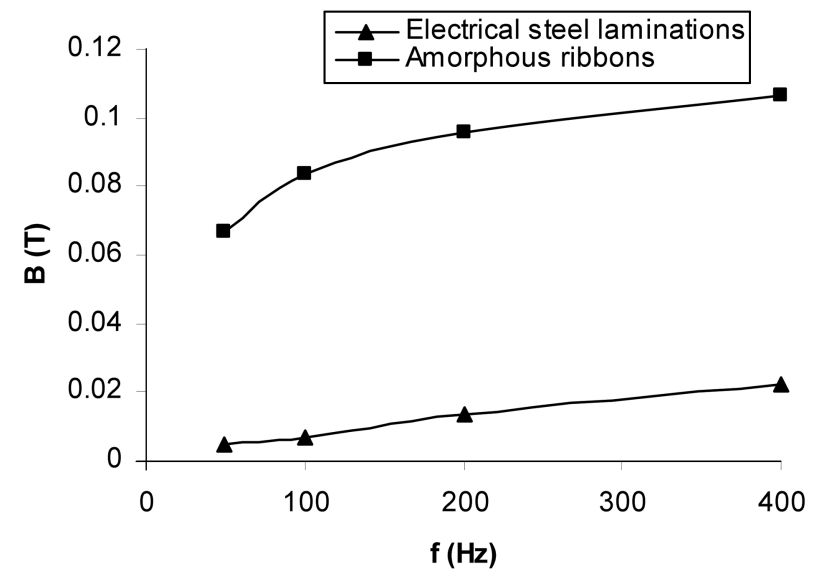

Fig. 5. Variation in flux density (B) with frequency (f) for search coil Z.

in flux density (B) were determined to be within $\pm 5 \%$ and $\pm 4 \%$ for the electrical steel and amorphous ribbons, respectively.

The flux density measured by search coil $\mathrm{Z}$ in the joint decreased by $96 \%$ for the electrical steel laminations and by $81 \%$ for the amorphous ribbons. The flux density measured by search coil Y (when compared with that measured by search coil X) increased by $38 \%$ for the electrical steel laminations and by $22 \%$ for the amorphous ribbons. Calculations were done by averaging the results for 50 , 100, 200, and $400 \mathrm{~Hz}$.

Electrical steel laminations and amorphous ribbons were modeled separately by using ANSYS ${ }^{\mathrm{TM}}$ to support the experimental data with a numerical method. The flux distributions computed by the FEA at three nominal densities for electrical steel laminations and at two nominal densities for amorphous ribbons are shown in Figs. 6 and 7 , respectively. These results indicate that when the permeability in the closed magnetic paths differs in different media, the flux changes direction toward the new lowest magnetic path length and then moves to the next layer with increasing magnetic flux density. This is confirmed by the measured data. The $2 \mathrm{D}$ results and measurements agree within $90 \%$.

\section{Conclusions}

The magnetic flux distributions predicted by FEA corresponded adequately with the measured values for both electrical steel laminations and amorphous ribbons.

Because of the low saturation magnetization of amorphous ribbons, the flux density measured by search coil Y in the electrical steel is higher than that of the amorphous ribbons (Fig. 4). We also confirmed from the FEA results 


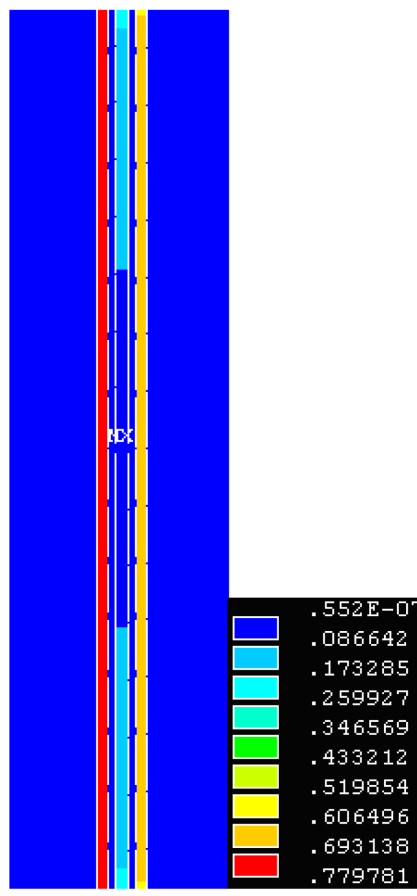

(a)

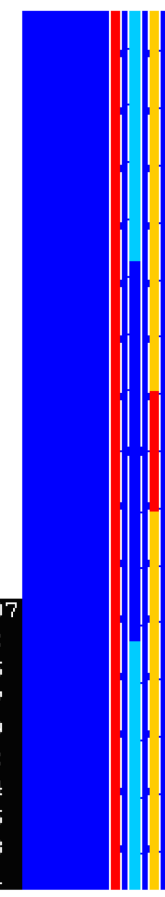

(b)

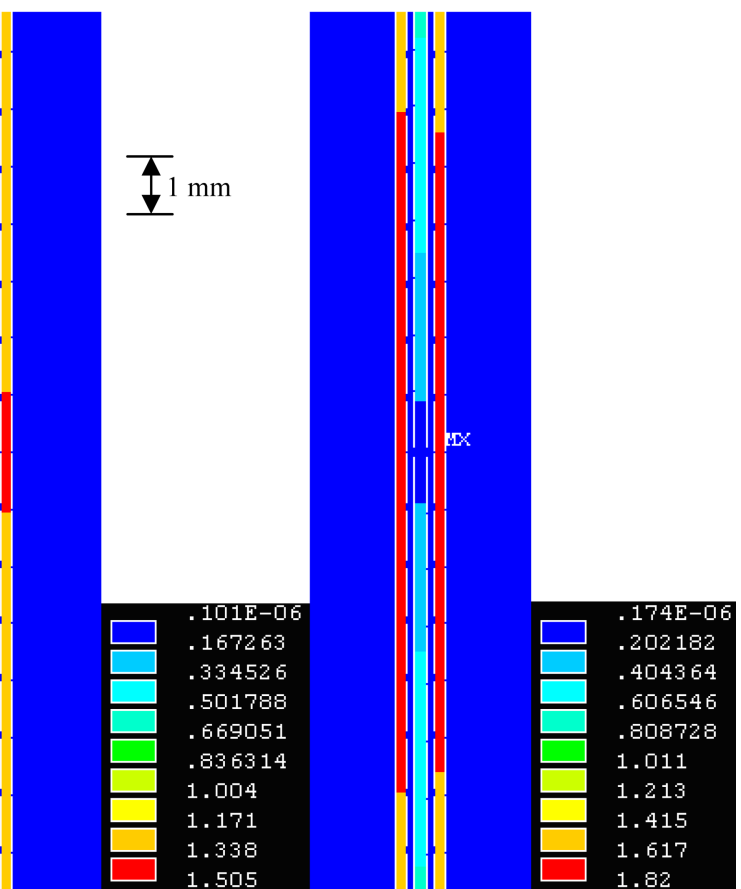

(c)

Fig. 6. Calculated flux distribution along laminations 1 to 4 at $100 \mathrm{~Hz}$ and (a) 0.5 , (b) 1.0 , and (c) $1.5 \mathrm{~T}$ for electrical steel laminations.

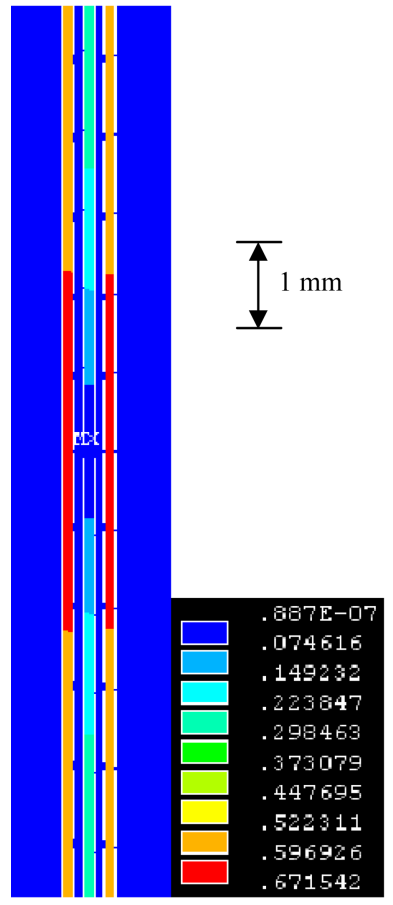

(a)

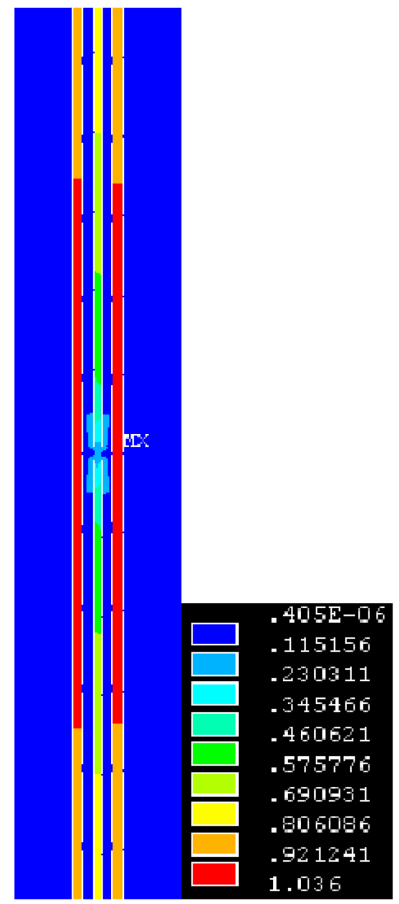

(b)

Fig. 7. Calculated flux distribution along ribbons 1 to 4 at 100 $\mathrm{Hz}$ and (a) 0.5 and (b) $0.9 \mathrm{~T}$ for amorphous ribbons.

that the flux density in the amorphous ribbons above and below the air gap begins to approach saturation (Fig. 7).

When the frequency values increased from 50 to 400 $\mathrm{Hz}$, the flux density measured by search coil $\mathrm{Y}$ increased in the amorphous ribbons and decreased in the electrical steel laminations (Fig. 4). On the other hand, the flux density measured by search coil $\mathrm{Z}$ in the amorphous ribbons was higher than that in the electrical steel (Fig. 5). The reasons lie in the permeability, electrical resistance, and isotropy of the materials. The amorphous ribbons provide more opportunities for magnetic flux transitions than electrical steel. Therefore, at high frequencies, the use of amorphous ribbons can yield more efficient transformers.

\section{References}

[1] M. A. Jones and A. J. Moses, IEEE Trans. Magn. 10, 321 (1974).

[2] S. Erdem, N. Derebasi, and A. Moses, J. Magn. Magn. Mater. 304, e807 (2006).

[3] C. Lee and H. Jung, IEEE Trans. Magn. 36, 693 (2000). 